\title{
Transmission and Reflection by a Parallel Wire Grid
}

\author{
Martin T. Decker
}

(February 19, 1959)

\begin{abstract}
A comparison is made at $\mathrm{X}$-band frequencies of the theoretical and measured transmission and reflection coefficients for a parallel wire grid. The methods used are applicable to the measurements of these factors for various building materials.
\end{abstract}

\section{Introduction}

As a preliminary phase in the measurement of the attenuating properties of various inhomogeneous materials and structures, tests have been made with an obstacle having electrical behavior which lends itself to calculation. These data will then be useful in evaluating the validity of certain test configurations. A planar grid of parallel wires was chosen as a suitable obstacle. The transmission and reflection of electromagnetic waves by such a grid have been treated in various papers, and solutions have been presented for both oblique angles of incidence and arbitrary polarization of plane waves. ${ }^{1,2} \mathrm{~A}$ comparison of one of these solutions with experiment has been made at frequencies near $9 \mathrm{kMc}$.

\section{Calculation of Grid Transmission and Reflection}

Reflection from a parallel wire grid mav be calculated according to the method given by Wait. ${ }^{1}$ The coordinate system used is shown in figure 1. Wait's paper treats the grid as infinite in the $y$-z plane, with the incident energy in the form of a plane wave with arbitrary polarization and angle of incidence. A special case is taken here in which the incident wavefront is a vertical plane, vertically polarized (electric vector parallel to the grid wires) and is incident on the grid at some angle $\theta$ measured in the $x-y$ plane.

In this special case, the reflection coefficient is given by

$$
R=\frac{-1}{1+2 Z_{g} / K_{0}}
$$

where $Z_{g}$ is the equivalent shunt impedance of the grid, $K_{g}$ is the intrinsic impedance of the incident wave in the direction normal to the grid and the ratio is given by

$$
Z_{g} / K_{0}=i\left(\frac{d}{\lambda}\right) \cos \theta\left[\ln \frac{d}{2 \pi \alpha}+F\left(\frac{d}{\lambda}, \theta\right)\right]+\cos \theta Z_{i} \frac{d}{\eta_{0}},
$$

\footnotetext{
1 J. R. Wait, Reflection at arbitrary incidence from a parallel wire grid, Appl. Sci. Research, B. 4, 393 (1954-5).
}

2 G. G. MacFarlane, Proc. Inst. Elec. Engrs. (London) Pt. IIIA 93, 1523 (1946)

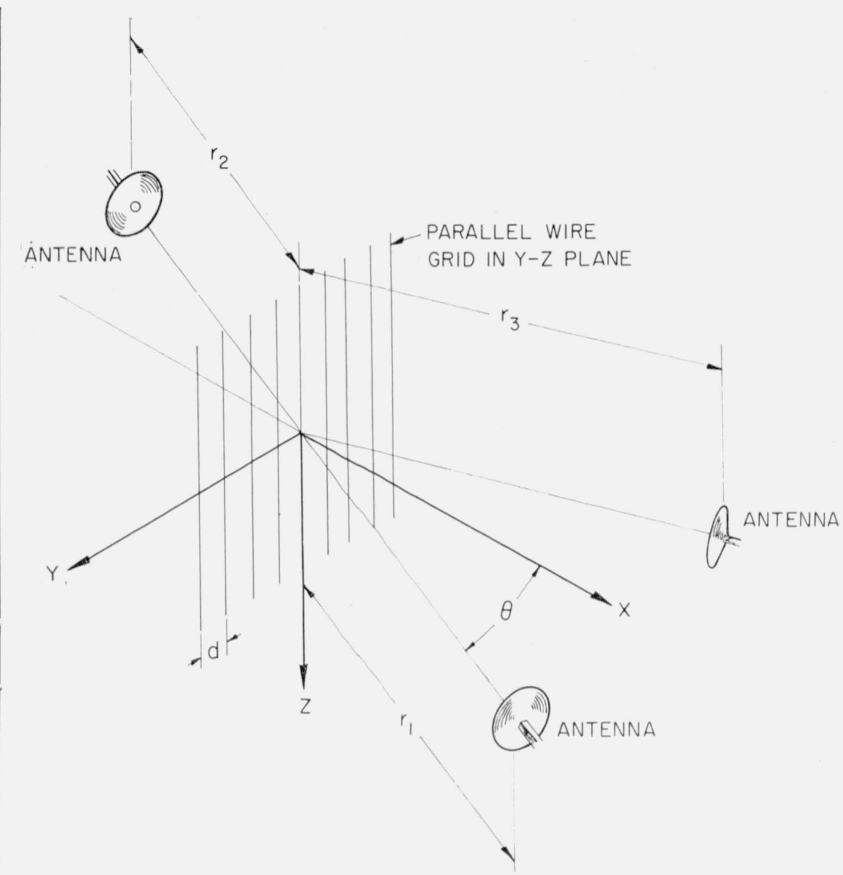

Figure 1. Coordinate system for parallel wire grid.

where

$d=$ spacing between adjacent wires.

$\lambda=$ wavelength.

$\alpha=$ radius of wires in the grid.

$F\left(\frac{d}{\lambda}, \theta\right)$ is a correction factor which has been evaluated by MacFarlane. ${ }^{2}$ A more general form is given by Wait. ${ }^{1}$ $\cos \theta Z_{i} \frac{d \text { may be considered a correction factor }}{\eta_{0}}$ involving the internal impedance $Z_{i}$ of the wire. It is sufficiently small to be neglected in this case.

With the restrictions that $d>>\alpha$ and that $\lambda>2 d$, $R$ may be evaluated for the conditions of the experiment.

$R^{2}$ is then the proportion of energy reflected from the grid (reflectivity). Assuming no losses in the grid, the fraction of energy transmitted (transmissivity) is given by

$$
T^{2}=1-R^{2} \text {. }
$$




\section{Grid Description}

The parallel wire grid used in these experiments consisted of 75 copper wires (1.02 $\mathrm{mm}$ diam) spaced $1.5 \mathrm{~cm}$ on centers, making a total width of $111 \mathrm{~cm}$. The wires were mounted on a frame approximately $3-\mathrm{m}$ square as shown in figure 2 . The frequencies used were 8.8 to $9.6 \mathrm{kMc}$, so that $\lambda$ varies over the range of $2.08 d$ to $2.27 d$. In this case the ratio $d / a$ is approximately 15 . The wires were long enough so that shorting or opening their ends did not affect the transmitting or reflecting properties of the grid.

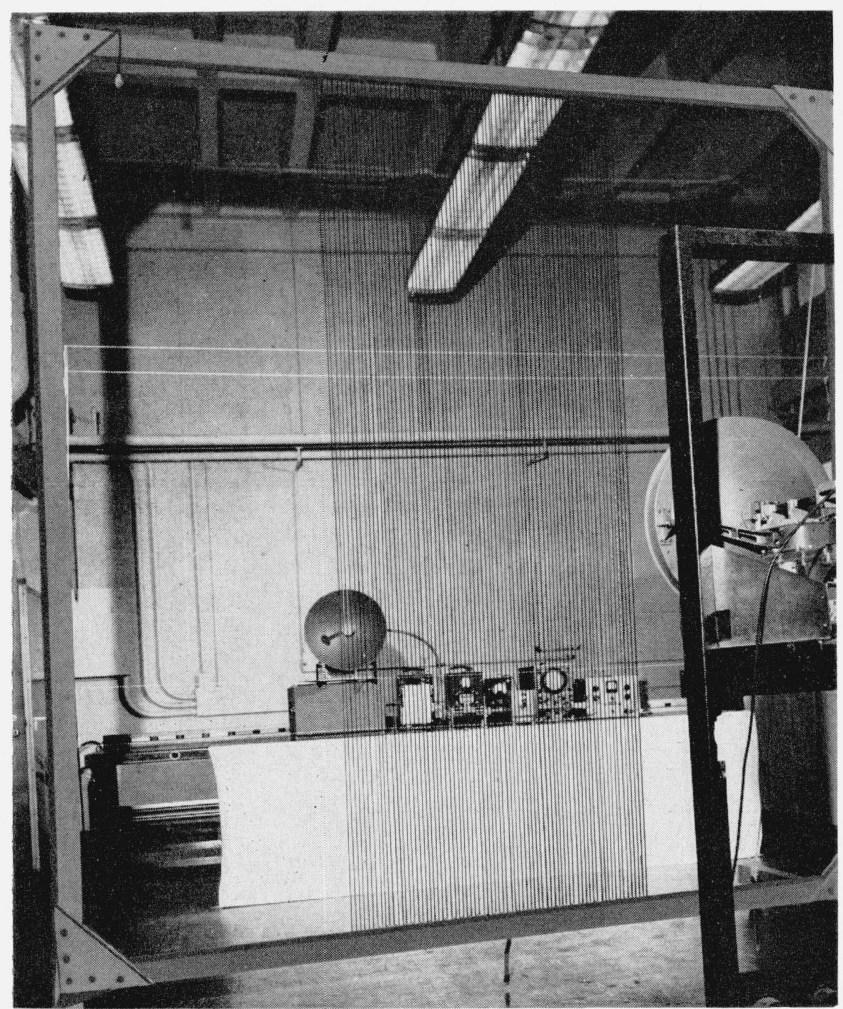

FIGURE 2. Transmission through a parallel wire grid.

\section{Equipment}

The measurements were made at X-band frequencies (8.8 to $9.6 \mathrm{kMc}$ ). A pulsed reflex klystron is used as a power source, with a ferrite isolator to minimize effects of the rest of the system on the power output and frequency of the source. The energy is fed through a frequency measuring device, precision attenuator and then through flexible waveguide to the transmitting antenna. Transmitting and receiving antennas are 18 -in. parabolic reflectors. Slide-screw tuners are used at both antennas to provide a good impedance match, since reflections from the system terminals complicate the measurement process. These reflections are particularly bothersome when the transmitted wave is normally incident to an intervening obstacle, and techniques to eliminate their effect must be employed. Received energy is detected by the simple arrangement of a crystal in the waveguide at the receiving antenna. The detected signal (1,000 cps) is brought to an a-c amplifier and the output of this amplifier is rectified and fed to the recording meter.

The precision attenuator provides the standard of relative power measurement. The change in attenuation required to produce a given indication at the receiving terminal with and without an intervening obstacle is used to determine the power transmission coefficient of the obstacle. This procedure eliminates the need for assumptions of specific crystal characteristics at various operating levels. Power reflection coefficients are measured in a similar manner, comparing the power reflected from a surface to the power received over a direct transmission path of equivalent length.

The directional pattern of the main lobe of the 18-in. parabolic antennas was measured and the results are shown in figure 3 . Here a relative power curve (in decibels) is plotted as a function of the H-plane angle from the antenna centerline, the apex of this angle being taken in the plane of the edge of the reflector. These measurements were made at a distance of 172 wavelengths $(5.5 \mathrm{~m})$.

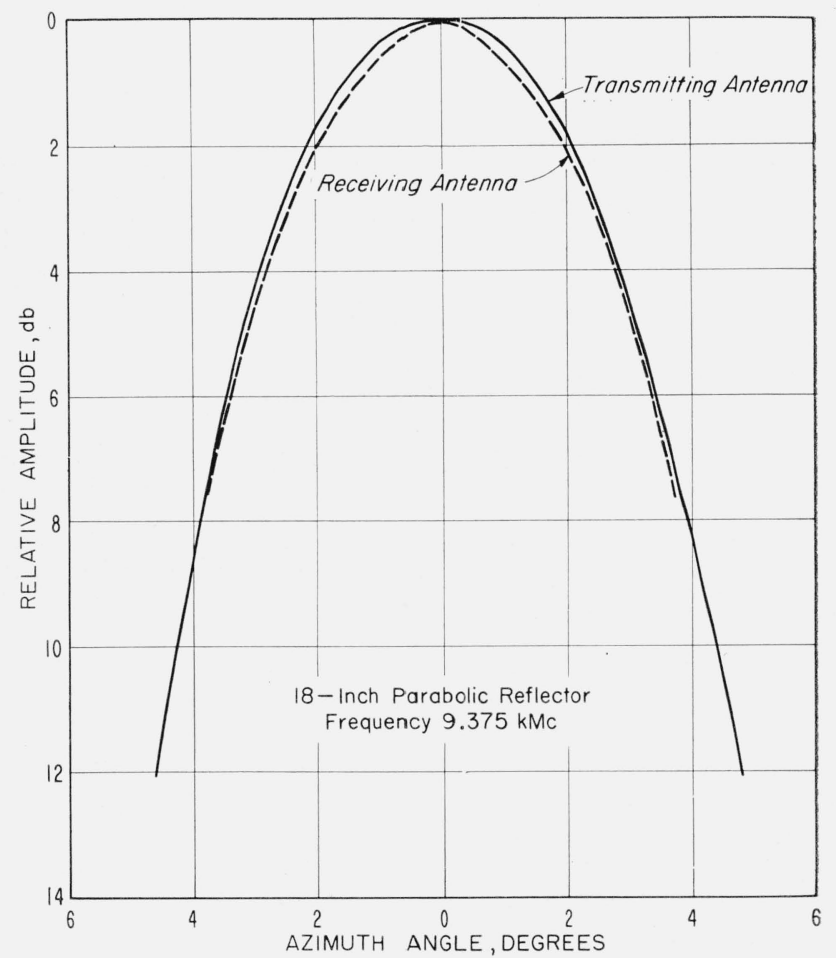

FIGURE 3. Horizontal antenna patterns for vertical polarization.

\section{Polarization}

Energy incident with the electric vector perpendicular to the wires of the grid should not excite currents of any appreciable magnitude in the wires and hence should not be reflected. This property was clearly demonstrated by experiment, the grid being completely transparent to horizontal polarization. This also indicates that the radiation from the antennas is linearly polarized. 


\section{Diffraction Effects}

An indication of the grid size necessary to make edge effects negligible was obtained by moving the grid into the path as shown in figure 4, while observing simultaneously the transmitted energy. The initial rise in transmission above the free space value is due to diffraction as the edge of the grid approaches the path. When the edge of the grid has moved past the path centerline a distance of about $30 \mathrm{~cm}$ the transmission value becomes practically constant. The vertical bars at each of the measured points indicate the total range of variation of the data as the distance between transmitting and receiving antennas is varied from 4.5 to $7.5 \mathrm{~m}$. A total minimum grid width of $60 \mathrm{~cm}$ would then be necessary for transmission measurement. This comment on grid size is applicable to an obstacle having attenuation comparable to the 1 to $2 \mathrm{db}$ of the grid. If the attenuation in the obstacle were much higher the relative magnitude of diffracted energy could be greater.

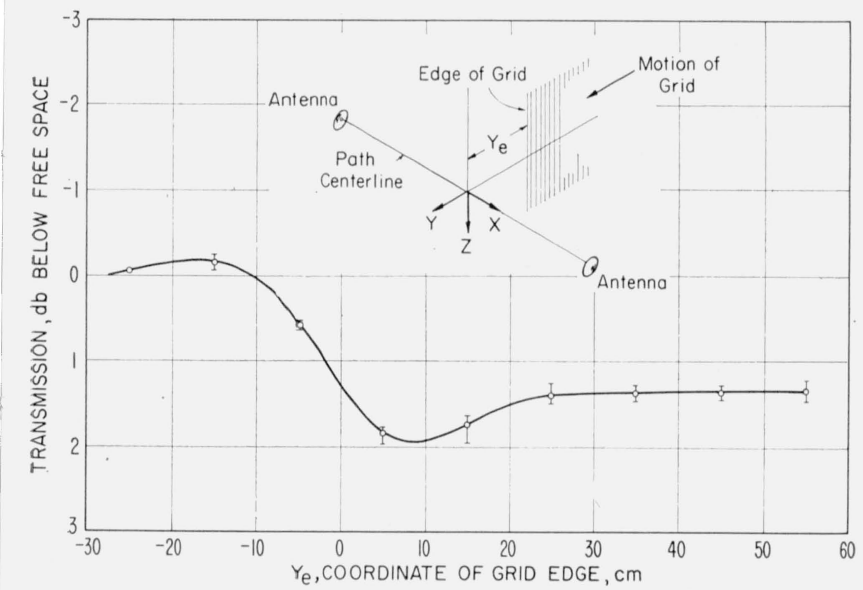

FIgURE 4. Transmission through a parallel wire grid as grid is moved into path of antennas.

\section{Grid Transmission}

A series of measurements of transmission through the $111-\mathrm{cm}$ grid (1.5-cm spacing) were made at different frequencies and angles of incidence. As an example, figure 5 shows the energy transmitted through the grid (relative to transmission with no obstruction between the antennas) for a wavelengtb of $3.2 \mathrm{~cm}(9.375 \mathrm{kMc})$ and at angles of incidence up to $60^{\circ}$. The antennas were separated $5.5 \mathrm{~m}$ with the grid at the center of the path. The curve marked "calculated" was obtained by calculating the reflection coefficient as indicated previously, then assuming no loss in the grid. A series of these curves at different frequencies in the X-band is shown in figure 6. Here the data of figure 5 appear as the fourth set of curves at $9.375 \mathrm{kMc}$. The relation between transmission and angle of incidence is shown by each pair of curves (one measured and one calculated) while an indication of the transmission versus frequency at a given angle of incidence can be obtained by connecting corresponding angle-of-incidence points on each of the curves. The agreement of theory and

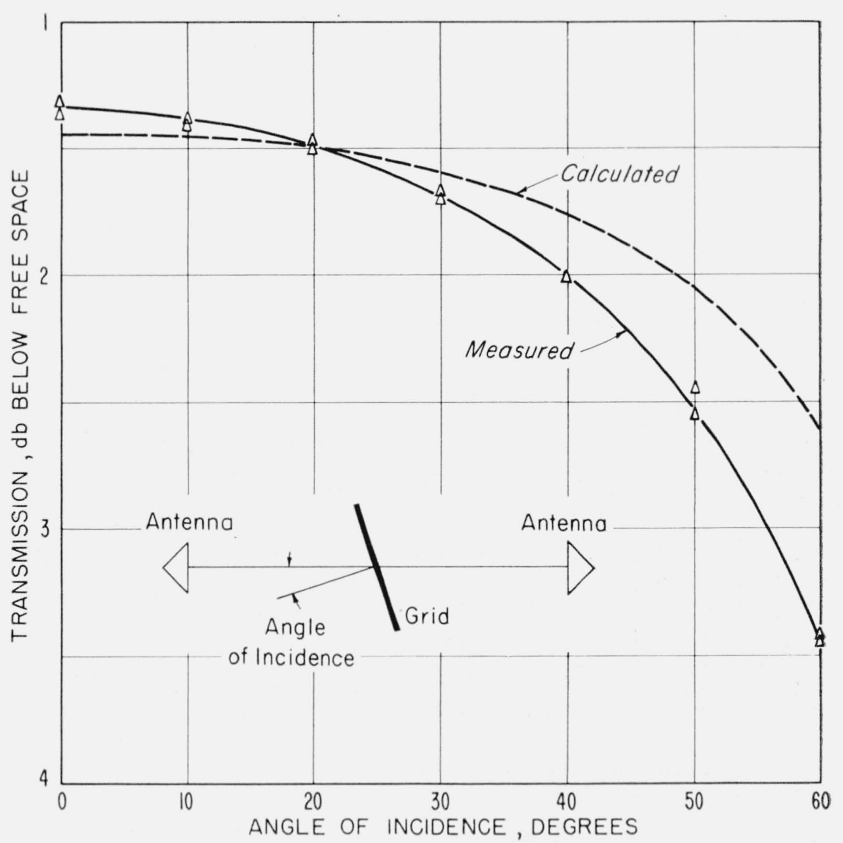

FIGURE 5. Transmission through a parallel wire grid as a function of angle of incidence.

measurement is fairly good at the lower angles of incidence, both with respect to angle of incidence and frequency. Discrepancy would be expected at the higher angles of incidence since at $60^{\circ}$, for example, the projection of the grid on a plane perpendicular to the path is $55 \mathrm{~cm}$, corresponding to the $27-\mathrm{cm}$ position of figure 4, where the projected grid size is becoming too small to agree with the infinite grid calculation. The lower transmission (higher reflection) also makes the diffracted energy relatively more important at these angles of incidence. It should also be noted that the calculation is made for a plane incident wave, while this condition is not completely met in the measurement.

\section{Grid Reflection}

Reflected and transmitted energy as a function of angle of incidence are shown in figure 7 . The percent of reflected energy (reflectivity) was measured at a wavelength of $3.2 \mathrm{~cm}$ over a range of angles from $7^{\circ}$ to $47^{\circ}$. Corresponding transmissivity data are shown for $0^{\circ}$ to $60^{\circ}$ incidence. The calculated curve may be considered to be either reflectivity or transmissivity on the assumption that there are no losses in the grid. The gap between the two measured curves is an indication of the portion of energy either lost in the grid itself or scattered in the directions other than the principal transmitted and reflected paths. This amounts to about 3 percent of the total energy for low angles of incidence, increasing to about 10 percent at the largest angle at which reflection measurements were made. This method does not separate the absorbed and scattered energy and hence is more effective in determining absorption from materials of fairly uniform composition and with plane surfaces. 


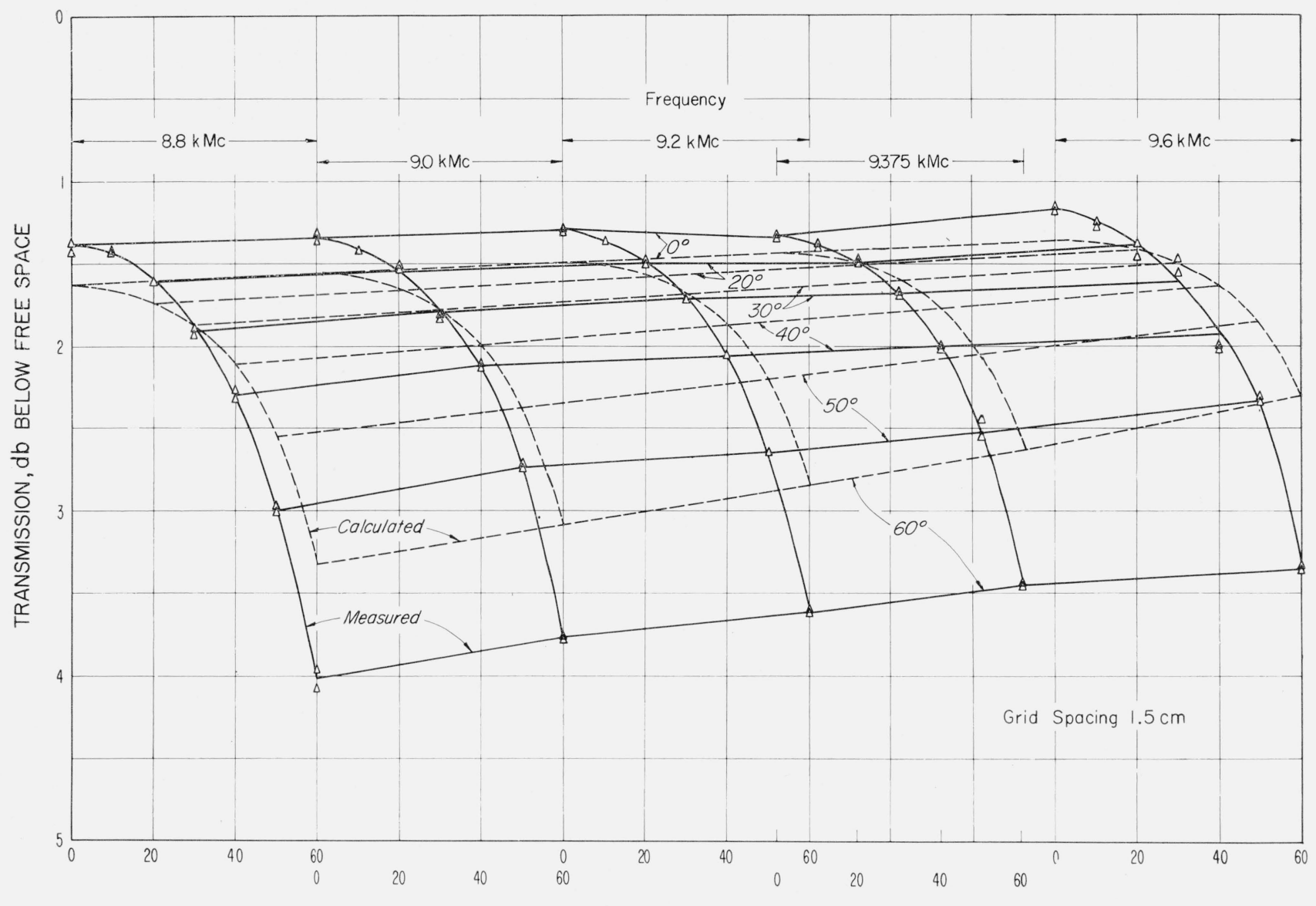

ANGLE OF INCIDENCE, DEGREES

FiguRE 6. Transmission through a parallel wire grid as a function of angle of incidence and frequency.

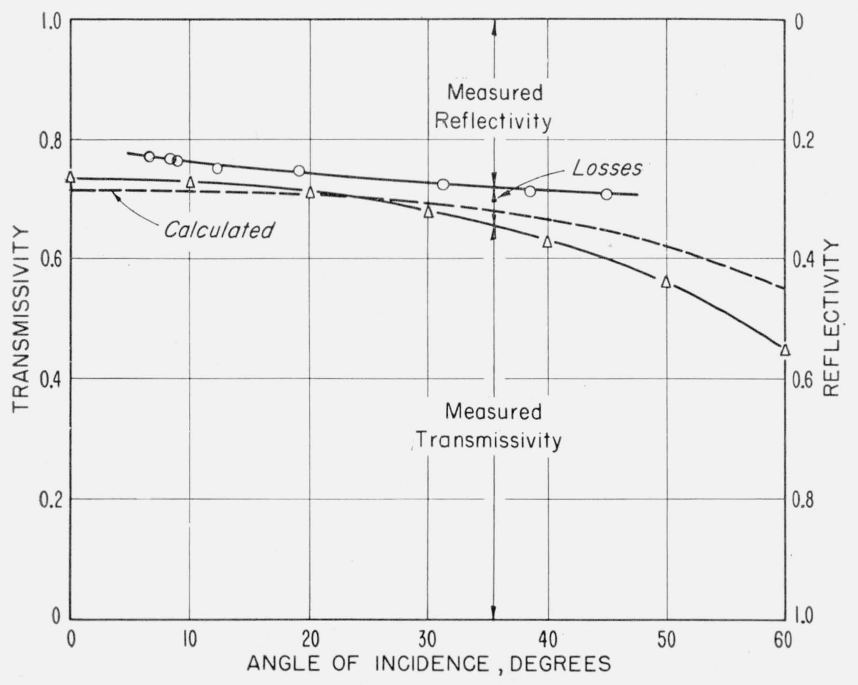

Figure 7. Proportion of energy transmitted and reflected by a parallel wire grid.

\section{Conclusion}

Transmission and reflection properties of a homogeneous material may often be determined from a knowledge of the dielectric constant and conductivity. If, however, the inhomogeneity of the material or the physical configuration of the material or radiating elements make calculation difficult, resort may be had to measurement. The agreement in theory and measurement obtained here indicates the degree to which the experimental configuration may be used in studying the properties of inhomogeneous structures. These methods are currently being applied over a wide frequency range in a study of various building materials and structures. The results may then be applied to cases in which it is impossible to avoid the presence of obstructing materials in an actual propagation path.

\section{Boulder, Colo. (Paper 63D1-10).}

